\title{
Consensus under general convexity
}

\author{
S. Emre Tuna and Rodolphe Sepulchre
}

\begin{abstract}
A method is proposed to characterize contraction of a set through orthogonal projections. For discrete-time multi-agent systems, quantitative estimates of convergence (to a consensus) rate are provided by means of contracting convex sets. Required convexity for the sets that should include the values that the transition maps of agents take is considered in a more general sense than that of Euclidean geometry.
\end{abstract}

\section{INTRODUCTION}

A multi-agent system is a collection of subsystems (called agents) each of which updates itself according (only) to the information it gathers from some of the other agents, that is, from its neighbors. Generally, the set of neighbors for each agent is subject to change in time; and this change naturally occurs with respect to the dynamics of the system. It is however, generally, too complicated to study a multiagent system in such precision especially when the number of agents is large [1]. Hence, usually the variation of the neighbor sets (i.e. variation of communication topology) is detached from the dynamics of the system by assumption Recently, it has been an active direction for researchers to study the effect of switching communication topologies on some common task to be accomplished, (i.e. reaching a consensus) by the agents composing the system. Among related applications are formation control, synchronization of coupled oscillators, and distributed sensor fusion in sensor networks; see, for instance, [2], [3], [4], respectively. We refer the reader to the recent survey [5] for details and a myriad of references.

Two particular papers to which the current work is closely related are [6] and [7]. Moreau shows in [6] that states of all agents evolve toward a consensus by converging to a common point if the following assumptions hold:

(a) the state of an agent at the next time step is in the (relative) interior of the convex hull of the set comprising the current state of that agent and its neighbors, and

(b) the graph describing the communication topology is uniformly connected over an interval.

His work is generalized in [7] where the convexity property in (a) is relaxed. The relaxation allows working with sets that are not necessarily convex but are transformable (via some invertible map) to convex sets. Both [6] and [7] establish only qualitative convergence results.

This paper presents research results of the Belgian Network DYSCO (Dynamical Systems, Control, and Optimization), funded by the Interuniversity Attraction Poles Programme, initiated by the Belgian State, Science Policy Office. The scientific responsibility rests with its authors. Authors are with the Department of Electrical Engineering and Computer Science, Montefiore Institute, University of Liege, 4000 Liege, Belgium. tuna@montefiore.ulg.ac.be, r.sepulchredulg.ac.be
Our purpose in this paper is to study quantitative aspects of convergence to a consensus [8] in a general setting. To attain quantitativeness, we impose on each subsystem that its state at the next time instant is in a contraction of the convex hull of the set comprising the current state of that subsystem and its neighbors. Our idea of contraction is based on orthogonal projections. Since it is straightforward how to contract a line segment, we have chosen to define contraction of an arbitrary convex set as its largest subset whose projection onto an arbitrary line is always within the contraction of the line segment that is the projection of the set to be contracted onto the same line. We exploit class- $\mathcal{K}$ functions to characterize contraction. To attain generality, we consider convexity in metric spaces more general than the standard Euclidean space, where line segments are replaced by geodesic segments but preserving the very essential property of their counterparts in $\mathbb{R}^{n}$ : being the unique shortest path between two points. In that respect, the generalization of the analysis here is seemingly different from the generalization in [7], but we defer comparison for later.

As the main contribution of this paper, we provide (in Theorem 3) an upperbound on the decay of the diameter of the set comprising the states of the agents via a class$\mathcal{K} \mathcal{L}$ function which we explicitly express in terms of the number of agents, the length of the interval over which the communication graph is connected, and a class- $\mathcal{K}$ function characterizing contraction. We also show that whenever this class- $\mathcal{K}$ function is linear, the convergence is exponential; and that for a multi-agent system that is a switched linear system with some convexity property (cf. [9]), contraction function, which turns out to be global and linear, can be explicitly computed in terms of the system parameters.

The rest of the paper is organized as follows. In the next section we provide notation, introduce contraction (which will be the key element for our results), and expose the unfamiliar reader to $\mathrm{CAT}(0)$ spaces (the class of metric spaces where we consider convexity in generality) by giving basic definitions and results. In Section III we describe the system. In Section IV we give stability results. Section V is where we provide our main results, namely, we present an algorithm to construct a class- $\mathcal{K} \mathcal{L}$ function and show that the convergence to a consensus can be characterized by it. Before we conclude, we present two examples: a linear system to display that basic cases are covered by the presented framework; and a loglinear system to emphasize that certain nonlinear systems can be studied in a natural way under general convexity. 


\section{PRELIMINARIES}

Nonnegative integers are denoted by $\mathbb{N}$, nonnegative reals by $\mathbb{R}_{\geq 0}$. Euclidean norm is denoted by $|\cdot|$. A function $\alpha$ : $\mathbb{R}_{\geq 0} \rightarrow \mathbb{R}_{\geq 0}$ is said to belong to class- $\mathcal{K}(\alpha \in \mathcal{K})$ if it is zero at zero, continuous, and strictly increasing. A function $\beta$ : $\mathbb{R}_{\geq 0} \times \mathbb{R}_{\geq 0} \rightarrow \mathbb{R}_{\geq 0}$ is said to belong to class- $\mathcal{K} \mathcal{L}(\beta \in \mathcal{K} \mathcal{L})$ if for each fixed $t, \beta(\cdot, t)$ is zero at zero, nondecreasing, and $\lim _{s \rightarrow 0^{+}} \beta(s, t)=0$; and for each fixed $s, \beta(s, \cdot)$ is nonincreasing and $\lim _{t \rightarrow \infty} \beta(s, t)=0$. Function id is such that $\operatorname{id}(s)=s$ for all $s \geq 0$. For $\gamma: \mathbb{R}_{\geq 0} \rightarrow \mathbb{R}_{\geq 0}$ and $k \in \mathbb{N}, \gamma^{k+1}(\cdot)=\gamma\left(\gamma^{k}(\cdot)\right)$ where $\gamma^{0}=$ id. Given two functions $\alpha_{1}, \alpha_{2}: \mathbb{R}_{\geq 0} \rightarrow \mathbb{R}_{\geq 0}$, we write $\alpha_{1} \leq \alpha_{2}$ to imply $\alpha_{1}(s) \leq \alpha_{2}(s)$ for all $s \geq 0$. (Meaning of $\alpha_{1}<\alpha_{2}$ should be obvious.) Given a map $f$ with domain $D$ and a set $C \subset$ $D$, we write $f(C)$ to denote the set $\{f(x): x \in C\}$. The definitions and results (except Proposition 1) of the following two subsections are borrowed from the book [10].

\section{A. Metric spaces, geodesics, and convexity}

Let $\mathbb{X}$ be a nonempty set. A metric on $\mathbb{X}$, or distance function on $\mathbb{X}$, is a map $d: \mathbb{X} \times \mathbb{X} \rightarrow \mathbb{R}_{\geq 0}$ with the following properties:

1) $d(x, y) \geq 0$ for every $x, y \in \mathbb{X}$, with equality holding if and only if $x=y$;

2) $d(x, y)=d(y, x)$ for every $x, y \in \mathbb{X}$; and

3) $d(x, y)+d(y, z) \geq d(x, z)$ for every $x, y, z \in \mathbb{X}$.

A metric space is a pair $(\mathbb{X}, d)$, where $d$ is a metric on $\mathbb{X}$. A metric space is said to be complete if every Cauchy sequence in it is convergent. Given a subset $X$ of $\mathbb{X}$, the diameter of $X$ is defined as $\operatorname{diam} X:=\sup _{x, y \in X} d(x, y)$. Set $X$ is said to be bounded if diam $X<\infty$. Given $\eta=\left(\eta_{1}, \eta_{2}, \ldots, \eta_{p}\right)$ and $\xi=\left(\xi_{1}, \xi_{2}, \ldots, \xi_{p}\right)$, both in $\mathbb{X}^{p}$, we let $d(\eta, \xi):=$ $\max _{i} d\left(\eta_{i}, \xi_{i}\right)$.

Let $(\mathbb{X}, d)$ be a metric space. A geodesic path joining $x \in \mathbb{X}$ to $y \in \mathbb{X}$, or geodesic from $x$ to $y$, is a map $c$ from a closed interval $[0, \ell] \subset \mathbb{R}$ to $\mathbb{X}$ such that $c(0)=x, c(\ell)=y$, and $d\left(c\left(t_{1}\right), c\left(t_{2}\right)\right)=\left|t_{1}-t_{2}\right|$ for all $t_{1}, t_{2} \in[0, \ell]$. The image of $c$ is called a geodesic segment. We will use $[x, y]$ to denote a geodesic segment which is the image of a geodesic from $x$ to $y$.

Let $I \subset \mathbb{R}$ be an interval. A map $c: I \rightarrow \mathbb{X}$ is said to be a constant speed geodesic if there exists a constant $\lambda$ such that $d\left(c\left(t_{1}\right), c\left(t_{2}\right)\right)=\lambda\left|t_{1}-t_{2}\right|$ for all $t_{1}, t_{2} \in I$.

A geodesic line in $\mathbb{X}$ is a map $c: \mathbb{R} \rightarrow \mathbb{X}$ such that $d\left(c\left(t_{1}\right), c\left(t_{2}\right)\right)=\left|t_{1}-t_{2}\right|$ for all $t_{1}, t_{2} \in \mathbb{R}$. The image of $c$ will also be called a geodesic line when there is no risk of ambiguity.

A metric space $(\mathbb{X}, d)$ is said to be uniquely geodesic if there is exactly one geodesic from $x$ to $y$ for all $x, y \in \mathbb{X}$. For a uniquely geodesic metric space $(\mathbb{X}, d)$, a set $C \subset \mathbb{X}$ is said to be convex if it contains all geodesic segments $[x, y]$ for all $x, y \in C$. Given a set $X \subset \mathbb{X}$, its convex hull, denoted conv $X$, is the intersection of all convex sets containing $X$.

Proposition 1 Let $(\mathbb{X}, d)$ be a uniquely geodesic metric space. Then for $X, Y \subset \mathbb{X}$ we have the following:

1) $X \subset \operatorname{conv} X$; and
2) $X \subset Y$ implies conv $X \subset \operatorname{conv} Y$.

\section{B. Comparison triangles and $\mathrm{CAT}(0)$ spaces}

A geodesic triangle $\triangle$ in a metric space $(\mathbb{X}, d)$ is the union of three geodesic segments $\left[v_{1}, v_{2}\right],\left[v_{2}, v_{3}\right],\left[v_{3}, v_{1}\right]$ which pairwise join the points $v_{1}, v_{2}, v_{3} \in \mathbb{X}$. Given a triangle $\triangle\left(\left[v_{1}, v_{2}\right],\left[v_{2}, v_{3}\right],\left[v_{3}, v_{1}\right]\right)$, a triangle $\bar{\triangle}\left(\bar{v}_{1}, \bar{v}_{2}, \bar{v}_{3}\right)$ is said to be a comparison triangle in $\mathbb{R}^{2}$ if $\left|\bar{v}_{1}-\bar{v}_{2}\right|=$ $d\left(v_{1}, v_{2}\right),\left|\bar{v}_{2}-\bar{v}_{3}\right|=d\left(v_{2}, v_{3}\right)$, and $\left|\bar{v}_{3}-\bar{v}_{1}\right|=d\left(v_{3}, v_{1}\right)$. A point $\bar{x} \in\left[\bar{v}_{1}, \bar{v}_{2}\right]$ is said to be a comparison point for $x \in\left[v_{1}, v_{2}\right]$ if $\left|\bar{x}-\bar{v}_{1}\right|=d\left(x, v_{1}\right)$. Likewise for the comparison points on $\left[\bar{v}_{2}, \bar{v}_{3}\right]$ and $\left[\bar{v}_{3}, \bar{v}_{1}\right]$.

A metric space $(\mathbb{X}, d)$ is said to be a $\operatorname{CAT}(0)$ space if for each $\triangle \subset \mathbb{X}$ and its comparison triangle $\bar{\triangle}$, it holds that for all $x, y \in \triangle$ and their comparison points $\bar{x}, \bar{y} \in \bar{\triangle}$, $d(x, y) \leq|\bar{x}-\bar{y}|$.

Proposition 2 If $(\mathbb{X}, d)$ is a $\mathrm{CAT}(0)$ space, then it is uniquely geodesic.

Proposition 3 Let $(\mathbb{X}, d)$ be a $\mathrm{CAT}(0)$ space, and let $C$ be a convex subset of $\mathbb{X}$ which is complete in the induced metric. Then,

1) there exists a unique map $\wp: \mathbb{X} \rightarrow C$, named orthogonal projection, with property $d(x, \wp(x))=\inf _{y \in C} d(x, y)$ for all $x \in \mathbb{X}$

2) $\wp$ is continuous; and

3) $\wp$ does not increase distances, i.e. $d(\wp(x), \wp(y)) \leq$ $d(x, y)$ for all $x, y \in \mathbb{X}$.

Proposition 4 If $(\mathbb{X}, d)$ is a $\mathrm{CAT}(0)$ space, then given any pair of constant speed geodesics $c_{i}:[0,1] \rightarrow \mathbb{X}$, for $i=$ 1,2 , the following holds for all $t \in[0,1]$ :

$d\left(c_{1}(t), c_{2}(t)\right) \leq(1-t) d\left(c_{1}(0), c_{2}(0)\right)+t d\left(c_{1}(1), c_{2}(1)\right)$

Observe, as a consequence of Proposition 4, that for a $\mathrm{CAT}(0)$ space $(\mathbb{X}, d)$ we can write diam conv $X=\operatorname{diam} X$ for all $X \subset \mathbb{X}$.

\section{Contraction}

Let $(\mathbb{X}, d)$ be a complete $\operatorname{CAT}(0)$ space and $\mathcal{L}_{\mathbb{X}}$ denote the set of all geodesic lines in $\mathbb{X}$. Let us be given a geodesic line $L \in \mathcal{L}_{\mathbb{X}}$, a geodesic segment $S \subset L$ with an associated geodesic $c:[0, \ell] \rightarrow S$, and a class- $\mathcal{K}$ function $\omega$ satisfying $\omega \leq \mathrm{id} / 2$. Then $\omega$-contraction of $S$ along $L$ is the geodesic segment $c([\omega(\ell), \ell-\omega(\ell)])=: \operatorname{cont}(S, \omega, L)$.

For a geodesic line $L \in \mathcal{L}_{\mathbb{X}}$, let $\wp_{L}: \mathbb{X} \rightarrow L$ be the orthogonal projection onto $L$. Let $C$ be a closed, bounded, and convex subset of $\mathbb{X}$. Then $\omega$-contraction of $C$ is

$\operatorname{cont}(C, \omega):=$

$$
\left\{x \in C: \wp_{L}(x) \in \overline{\operatorname{cont}}\left(\wp_{L}(C), \omega, L\right), L \in \mathcal{L}_{\mathbb{X}}\right\} .
$$

\section{Directed graphs and connectedness}

A directed graph is a pair $(\mathcal{N}, \mathcal{A})$ where $\mathcal{N}$ is a nonempty finite set (of nodes) and $\mathcal{A}$ is a finite collection of pairs (arcs) $\left(n_{i}, n_{j}\right)$ with $n_{i}, n_{j} \in \mathcal{N}$. A path from $n_{1}$ to $n_{l}$ is a sequence of nodes $\left\{n_{1}, n_{2}, \ldots, n_{l}\right\}$ such that $\left(n_{i}, n_{i+1}\right)$ is an arc for $i \in\{1,2, \ldots, l-1\}$. A directed graph is said 
to be connected if it has a node to which there exists a path from every other node.

\section{SYSTEM DESCRIPTION}

Let $(\mathbb{X}, d)$ be a complete $\operatorname{CAT}(0)$ space. Consider the system of $p$ agents

$$
\begin{aligned}
\mathbf{x}_{1}^{+} & =\mathbf{f}_{1}(\mathbf{x}, g) \\
\mathbf{x}_{2}^{+} & =\mathbf{f}_{2}(\mathbf{x}, g) \\
& \vdots \\
\mathbf{x}_{p}^{+} & =\mathbf{f}_{p}(\mathbf{x}, g)
\end{aligned}
$$

where $\mathbf{x}_{i} \in \mathbb{X}$ is the state of the $i$ th agent, $g$ is a parameter taking its values from some set $\mathcal{G}, \mathbf{x}=\left(\mathbf{x}_{1}, \mathbf{x}_{2}, \ldots, \mathbf{x}_{p}\right) \in$ $\mathbb{X}^{p}, \mathbf{f}_{i}: \mathbb{X}^{p} \times \mathcal{G} \rightarrow \mathbb{X}$ for $i \in\{1,2, \ldots, p\}$, and $\mathbf{x}_{i}^{+}$ denotes $i$ th agent's state at the next time instant. A sequence $\left\{g_{0}, g_{1}, \ldots\right\}$ in $\mathcal{G}$ is denoted by $\mathbf{g}$. The solution of system (1) at time $k \in \mathbb{N}$, having started at the initial condition $\mathbf{x}$ and evolved under the influence of the sequence $\mathbf{g}$ is denoted by $\Phi(k, \mathbf{x}, \mathbf{g})$. Note that $\Phi(0, \mathbf{x}, \mathbf{g})=\mathbf{x}$. Likewise, $\Phi_{i}(k, \mathbf{x}, \mathbf{g})$ denotes the solution of the $i$ th agent. When we write $\{\mathbf{x}\}$ we will mean $\left\{\mathbf{x}_{1}, \mathbf{x}_{2} \ldots, \mathbf{x}_{p}\right\}$, i.e. a subset of $\mathbb{X}$.

Let $\mathcal{N}=\left\{n_{1}, n_{2}, \ldots, n_{p}\right\}$ be the set of nodes of some graph $(\mathcal{N}, \mathcal{A})$ where node $n_{i}$ represents the $i$ th agent. Then, given a set of arcs $\mathcal{A}$, extended neighbor set of $i$ th agent is $\mathbf{n}_{i}(\mathbf{x}, \mathcal{A}):=\left\{\mathbf{x}_{j}:\left(n_{i}, n_{j}\right) \in \mathcal{A}\right\} \cup\left\{\mathbf{x}_{i}\right\}$. For each $g \in \mathcal{G}$ there is an associated set of arcs $\mathcal{A}_{g}$ and hence an associated graph $\left(\mathcal{N}, \mathcal{A}_{g}\right)$. For $N \in \mathbb{N}$, let us let $\mathbf{G}_{N}$ denote the set of sequences $\mathbf{g}=\left\{g_{0}, g_{1}, \ldots\right\}$ such that for each $k_{0} \in \mathbb{N}$, the union $\left(\mathcal{N}, \cup_{k=k_{0}}^{k_{0}+N} \mathcal{A}_{g_{k}}\right)$ is connected.

Definition 1 Given a set $C \subset \mathbb{X}$ and a class- $\mathcal{K}$ function $\omega \leq \mathrm{id} / 2$, system (1) is said to be contractive on $C$ with $\omega$ if for all $\{\mathbf{x}\} \subset C, g \in \mathcal{G}$, and $i \in\{1,2, \ldots, p\}$

$$
\mathbf{f}_{i}(\mathbf{x}, g) \in \operatorname{cont}\left(\operatorname{conv} \mathbf{n}_{i}\left(\mathbf{x}, \mathcal{A}_{g}\right), \omega\right)
$$

and is said to be contractive if for each bounded set $C \subset \mathbb{X}$ there exists a class- $\mathcal{K}$ function $\omega \leq \mathrm{id} / 2$ such that system (1) is contractive on $C$ with $\omega$.

Let $\mathbb{E} \subset \mathbb{X}^{p}$ denote the set of equilibrium points which we define as $\mathbb{E}:=\left\{\mathbf{x} \in \mathbb{X}^{p}: \operatorname{diam}\{\mathbf{x}\}=0\right\}$. The following definition is borrowed from [7].

Definition 2 System (1) with respect to sequence set $\mathbf{G}$ is

1) stable if for each $\xi \in \mathbb{E}$ and $\varepsilon>0$ there exists $\delta>0$ such that $d(\mathbf{x}, \xi) \leq \delta$ and $\mathbf{g} \in \mathbf{G}$ imply $d(\Phi(k, \mathbf{x}, \mathbf{g}), \xi) \leq \varepsilon$ for all $k$.

2) bounded if for each $\xi \in \mathbb{E}$ and $\delta>0$ there exists $\varepsilon>0$ such that $d(\mathbf{x}, \xi) \leq \delta$ and $\mathbf{g} \in \mathbf{G}$ imply $d(\Phi(k, \mathbf{x}, \mathbf{g}), \xi) \leq \varepsilon$ for all $k$.

3) attractive if for each $\xi \in \mathbb{E}$ and $\varepsilon, \delta>0$ there exists $K \in \mathbb{N}$ such that $d(\mathbf{x}, \xi) \leq \delta$ and $\mathbf{g} \in \mathbf{G}$ imply the existence of $\eta \in \mathbb{E}$ such that $d(\Phi(k, \mathbf{x}, \mathbf{g}), \eta) \leq \varepsilon$ for all $k \geq K$.

4) asymptotically stable if it is stable, bounded, and attractive.

We end the section with the following assumptions which will henceforth hold on our metric space $(\mathbb{X}, d)$.
Standing Assumption 1 Let $L \in \mathcal{L}_{\mathbb{X}}$ be a geodesic line and $\wp_{L}: \mathbb{X} \rightarrow L$ be the orthogonal projection onto $L$. For all $x, y \in \mathbb{X}$ we have $\wp_{L}([x, y])=\left[\wp_{L}(x), \wp_{L}(y)\right]$.

Standing Assumption 2 Every geodesic segment lies on a geodesic line.

\section{AsYMPTOTIC STABILITY}

Here we provide the stability results for contractive systems. Similar results were established in [6] and later in [7] under different assumptions.

Theorem 1 Let system (1) be contractive. Then, for all $\{\mathbf{x}\} \subset \mathbb{X}$ and all sequences $\mathbf{g}$ in $\mathcal{G},\{\Phi(k, \mathbf{x}, \mathbf{g})\} \subset \operatorname{conv}\{\mathbf{x}\}$ for all $k \in \mathbb{N}$.

Proof. Let us be given $\mathbf{x}$ and $\mathbf{g}=\left\{g_{0}, g_{1}, \ldots\right\}$. For all $i \in\{1,2, \ldots, p\}$ and $k \in \mathbb{N}$ we can write

$$
\begin{aligned}
\Phi_{i}(k+1, \mathbf{x}, \mathbf{g}) & =\mathbf{f}_{i}\left(\Phi(k, \mathbf{x}, \mathbf{g}), g_{k}\right) \\
& \in \operatorname{cont}\left(\operatorname{conv} \mathbf{n}_{i}\left(\Phi(k, \mathbf{x}, \mathbf{g}), \mathcal{A}_{g_{k}}\right), \omega\right)
\end{aligned}
$$

for some $\omega \in \mathcal{K}$ since the system is contractive. By definition $\mathbf{n}_{i}\left(\Phi(k, \mathbf{x}, \mathbf{g}), \mathcal{A}_{g_{k}}\right) \subset\{\Phi(k, \mathbf{x}, \mathbf{g})\}$. Therefore, due to Proposition 1 , we can continue as

$$
\begin{aligned}
\Phi_{i}(k+1, \mathbf{x}, \mathbf{g}) & \in \operatorname{cont}\left(\operatorname{conv} \mathbf{n}_{i}\left(\Phi(k, \mathbf{x}, \mathbf{g}), \mathcal{A}_{g_{k}}\right), \omega\right) \\
& \subset \operatorname{conv} \mathbf{n}_{i}\left(\Phi(k, \mathbf{x}, \mathbf{g}), \mathcal{A}_{g_{k}}\right) \\
& \subset \operatorname{conv}\{\Phi(k, \mathbf{x}, \mathbf{g})\}
\end{aligned}
$$

which implies $\{\Phi(k+1, \mathbf{x}, \mathbf{g})\} \subset \operatorname{conv}\{\Phi(k, \mathbf{x}, \mathbf{g})\}$. Note that $\Phi(0, \mathbf{x}, \mathbf{g})=\mathbf{x}$. The result follows by induction.

Theorem 2 If system (1) is contractive, then, for all $N$, it is asymptotically stable with respect to set of sequences $\mathbf{G}_{N}$.

We defer the proof of Theorem 2 until after Theorem 3 of the next section.

\section{QUANTITATIVE CONVERGENCE ANALYSIS}

Algorithm 1 Given a triple $(\omega, p, N)$ where $\omega \leq \mathrm{id} / 2$ is a class- $\mathcal{K}$ function, $p \in \mathbb{N}_{\geq 1}$, and $N \in \mathbb{N}$, construct $\beta_{(\omega, p, N)} \in$ $\mathcal{K} \mathcal{L}$ through the following steps.

1) Let $\gamma_{1}: \mathbb{R}_{\geq 0} \rightarrow \mathbb{R}_{\geq 0}$ be such that

$$
\omega\left(s-\gamma_{1}(s)\right)=\gamma_{1}(s) \quad \forall s \geq 0 .
$$

(Note that $\gamma_{1} \in \mathcal{K}$ and $\gamma_{1} \leq \mathrm{id} / 3$.)

2) Define $\gamma_{2}: \mathbb{R}_{\geq 0} \rightarrow \mathbb{R}_{\geq 0}$ as

$$
\gamma_{2}(s):=s-\gamma_{1}^{(p-1)(N+1)}\left(\gamma_{1}(s) / 2\right) \quad \forall s \geq 0 .
$$

(Note that $5 \mathrm{id} / 6 \leq \gamma_{2}<\mathrm{id}$.)

3) If $\gamma_{2}$ is nondecreasing, define $\gamma_{3}(\cdot):=\gamma_{2}(\cdot)$; else let $I_{k}:=\left[2^{2 k-1}, 2^{2 k+1}\right]$ and $m_{k}:=$ $\inf \left\{m: m s \geq \gamma_{2}(s), s \in I_{k}\right\}$ for $k \in$ $\{\ldots,-1,0,1, \ldots\}$. Also let $\left|I_{k}\right|=2^{2 k+1}-2^{2 k-1}$. Then let $y_{k}^{+}:=\max \left\{m_{k} 2^{2 k+1}, m_{k+1} 2^{2 k+1}\right\}$ and $y_{k}^{-}:=\max \left\{m_{k} 2^{2 k-1}, m_{k-1} 2^{2 k-1}\right\}$. (Note that $y_{k}^{+}=$ $y_{k+1}^{-}$.) Then define $\gamma_{3}: \mathbb{R}_{\geq 0} \rightarrow \mathbb{R}_{\geq 0}$ as

$\gamma_{3}(s):=\left\{\begin{array}{cl}0 & \text { for } s=0 \\ y_{k}^{-}+\frac{y_{k}^{+}-y_{k}^{-}}{\left|I_{k}\right|}\left(s-2^{2 k-1}\right) & \text { for } s \in I_{k} .\end{array}\right.$ 
(Note that $\gamma_{3} \in \mathcal{K}$ and $\gamma_{2} \leq \gamma_{3}<$ id.)

4) Finally,

$$
\beta_{(\omega, p, N)}(s, t):=\gamma_{3}^{l}(s) \quad \forall s \geq 0
$$

for $t \in[(p-1)(N+1) l,(p-1)(N+1)(l+1)-1]$ and for $l \in \mathbb{N}$.

Algorithm 2 Given a triple $(w, p, N)$ where $0<w \leq 1 / 2$, $p \in \mathbb{N}_{\geq 1}$, and $N \in \mathbb{N}$, obtain the pair $(M, \sigma)$ as

$$
\begin{aligned}
M & :=\left(1-\frac{1}{2}\left(\frac{w}{1+w}\right)^{(p-1)(N+1)+1}\right)^{-1} \\
\sigma & :=\left(1-\frac{1}{2}\left(\frac{w}{1+w}\right)^{(p-1)(N+1)+1}\right)^{\frac{1}{(p-1)(N+1)}} .
\end{aligned}
$$

Lemma 1 Given a geodesic line $L \in \mathcal{L}_{\mathbb{X}}$ let $\wp_{L}: \mathbb{R} \rightarrow \mathbb{X}$ be the orthogonal projection onto $L$. Then, for all $X \subset \mathbb{X}$ we have $\wp_{L}(\operatorname{conv} X)=\operatorname{conv} \wp_{L}(X)$.

Proof. Proof is a direct consequence of Standing Assumption 1 and the definition of convexity.

Lemma 2 Let $\ell \geq 0, c:[0, \ell] \rightarrow \mathbb{X}$ be a geodesic, $L \in \mathcal{L}_{\mathbb{X}}$ be a geodesic line such that $c([0, \ell]) \subset L$ and $\omega \leq \mathrm{id} / 2$ be a class- $\mathcal{K}$ function. Let $\gamma_{1}: \mathbb{R}_{\geq 0} \rightarrow \mathbb{R}_{\geq 0}$ satisfy (3). Then, given a geodesic segment $S$ in $c([0, \ell])$ satisfying $S \supset$ $c([a, b])$ where $0 \leq a \leq \gamma_{1}(\ell) / 2$ and $\ell-\gamma_{1}(\ell) / 2 \leq b \leq \ell$ we have

$$
\overline{\operatorname{cont}}(S, \omega, L) \subset c\left(\left[\gamma_{1}(\ell), \ell-\gamma_{1}(\ell)\right]\right) .
$$

Proof. Without loss of generality we can take $S=c([a, b])$ since we can always redefine $a$ and $b$ within the allowed range. Note that $b-a \geq \ell-\gamma_{1}(\ell)$. We can write therefore

$$
\begin{aligned}
\overline{\operatorname{cont}}(S, \omega, L) & =c([a+\omega(b-a), b-\omega(b-a)]) \\
& \subset c\left(\left[a+\omega\left(\ell-\gamma_{1}(\ell)\right), b-\omega\left(\ell-\gamma_{1}(\ell)\right)\right]\right) \\
& \subset c\left(\left[\omega\left(\ell-\gamma_{1}(\ell)\right), \ell-\omega\left(\ell-\gamma_{1}(\ell)\right)\right]\right) \\
& =c\left(\left[\gamma_{1}(\ell), \ell-\gamma_{1}(\ell)\right]\right) .
\end{aligned}
$$

Hence the result.

Lemma 3 Let $\ell \geq 0, c:[0, \ell] \rightarrow \mathbb{X}$ be a geodesic, $L \in \mathcal{L}_{\mathbb{X}}$ be a geodesic line such that $c([0, \ell]) \subset L$ and $\omega \leq \mathrm{id} / 2$ be a class- $\mathcal{K}$ function. Let $\gamma_{1}: \mathbb{R}_{\geq 0} \rightarrow \mathbb{R}_{\geq 0}$ satisfy (3). Given $t \in[0, \ell]$ and a geodesic segment $S \subset c([0, \ell])$ satisfying $c(t) \in S$ we can write

$$
\overline{\operatorname{cont}}(S, \omega, L) \subset c\left(\left[\gamma_{1}(h), \ell-\gamma_{1}(h)\right]\right)
$$

where $h=\min \{t, \ell-t\}$.

Proof. Without loss of generality let $t=\min \{t, \ell-t\}$. Then suppose that the assertion is false. That implies that there exists $\bar{t} \in[0, \ell]$ satisfying either $\bar{t}<\gamma_{1}(t)$ or $\bar{t}>\ell-\gamma_{1}(t)$ such that $c(\bar{t}) \in \overline{\operatorname{con} t}(S, \omega, L)$ whence $c(\bar{t}) \in S$. Let us first consider the case $\bar{t}<\gamma_{1}(t)$ which implies $\bar{t}<t$ since $\gamma_{1}<$ id. Recall that $c(t) \in S$. Therefore there should exist $a \in[0, \bar{t}]$ and $b \in[t, \ell]$ such that $S=c([a, b])$. We then can write

$$
\begin{aligned}
\overline{\operatorname{cont}}(S, \omega, L) & =c([a+\omega(b-a), b-\omega(b-a)]) \\
& \subset c([\omega(b-a), \ell]) \\
& \subset c([\omega(t-\bar{t}), \ell]) \\
& \subset c\left(\left[\omega\left(t-\gamma_{1}(t)\right), \ell\right]\right) \\
& =c\left(\left[\gamma_{1}(t), \ell\right]\right)
\end{aligned}
$$

which contradicts with $c(\bar{t}) \in \overline{\operatorname{con}} \mathrm{t}(S, \omega, L)$. Now we look at the other case. Suppose $\bar{t}>\ell-\gamma_{1}(t)$ which implies $\bar{t}>t$ for $\gamma_{1} \leq \mathrm{id} / 3$ and $t \leq \ell / 2$. Therefore there should exist $a \in[0, t]$ and $b \in[\bar{t}, \ell]$ such that $S=c([a, b])$. We then can write, since $t \leq \ell / 2$,

$$
\begin{aligned}
\overline{\operatorname{cont}}(S, \omega, L) & =c([a+\omega(b-a), b-\omega(b-a)]) \\
& \subset c([0, \ell-\omega(b-a)]) \\
& \subset c([0, \ell-\omega(\bar{t}-t)]) \\
& \subset c\left(\left[0, \ell-\omega\left(\ell-\gamma_{1}(t)-t\right)\right]\right) \\
& \subset c\left(\left[0, \ell-\omega\left(\ell-\gamma_{1}(\ell / 2)-\ell / 2\right)\right]\right) \\
& =c\left(\left[0, \ell-\omega\left(\ell / 2-\gamma_{1}(\ell / 2)\right)\right]\right) \\
& =c\left(\left[0, \ell-\gamma_{1}(\ell / 2)\right]\right) \\
& \subset c\left(\left[0, \ell-\gamma_{1}(t)\right]\right)
\end{aligned}
$$

which contradicts with $c(\bar{t}) \in \overline{\operatorname{con}} \mathrm{t}(S, \omega, L)$.

Lemma 4 Let $C$ be a convex subset of $\mathbb{X}, \omega \leq \mathrm{id} / 2$ be a class- $\mathcal{K}$ function, and $N \in \mathbb{N}$. Suppose that system (1) is contractive on $C$ with $\omega$. Then for all $\{\mathbf{x}\} \subset C$ and $\mathbf{g} \in \mathbf{G}_{N}$ we have

$\operatorname{diam} \wp_{L}(\{\Phi(k, \mathbf{x}, \mathbf{g})\}) \leq \beta_{(\omega, p, N)}\left(\operatorname{diam} \wp_{L}(\{\mathbf{x}\}), k\right)$

for all geodesic lines $L \in \mathcal{L}_{\mathbb{X}}$ and $k \in \mathbb{N}$, where function $\beta_{(\omega, p, N)}$ is constructed according to Algorithm 1.

Proof. First we point out that, due to Proposition 1 and Theorem 1, the solution of system (1) stays in $C$ at all times if the initial condition lies in $C$. Let us be given $L \in \mathcal{L}_{\mathbb{X}}$, $\{\mathbf{x}\} \subset C$, and $\mathbf{g}=\left\{g_{0}, g_{1}, \ldots\right\} \in \mathbf{G}_{N}$. Then, for economic purposes, let us let $\phi_{i}^{k}:=\Phi_{i}(k, \mathbf{x}, \mathbf{g})$ for $i \in\{1,2, \ldots, p\}$ and $\phi^{k}:=\Phi(k, \mathbf{x}, \mathbf{g})$. Note that $\left\{\phi^{k}\right\} \subset C$ for all $k \in \mathbb{N}$. Since the system is contractive on $C$ with $\omega$, we can write by Lemma 1

$$
\begin{aligned}
\wp_{L}\left(\phi_{i}^{k+1}\right) & =\wp_{L}\left(\mathbf{f}_{i}\left(\phi^{k}, g_{k}\right)\right) \\
& \in \wp_{L}\left(\operatorname{cont}\left(\operatorname{conv} \mathbf{n}_{i}\left(\phi^{k}, \mathcal{A}_{g_{k}}\right), \omega\right)\right) \\
& \subset \overline{\operatorname{cont}}\left(\wp_{L}\left(\operatorname{conv} \mathbf{n}_{i}\left(\phi^{k}, \mathcal{A}_{g_{k}}\right)\right), \omega, L\right) \\
& =\overline{\operatorname{cont}}\left(\operatorname{conv} \wp_{L}\left(\mathbf{n}_{i}\left(\phi^{k}, \mathcal{A}_{g_{k}}\right)\right), \omega, L\right)
\end{aligned}
$$

for all $i$ and $k$. An implication of (4) is that $\wp_{L}\left(\phi_{i}^{k}\right) \in$ conv $\wp_{L}\left(\left\{\phi^{0}\right\}\right)$ for all $i$ and $k$. Hence, due to time invariance we can write

$$
\wp_{L}\left(\left\{\phi^{k+1}\right\}\right) \in \operatorname{conv} \wp_{L}\left(\left\{\phi^{k}\right\}\right) .
$$

Let $\ell=\operatorname{diam} \wp_{L}\left(\left\{\phi^{0}\right\}\right)$ and $c:[0, \ell] \rightarrow \mathbb{X}$ be the geodesic associated to geodesic segment conv $\wp_{L}\left(\left\{\phi^{0}\right\}\right)$. Note that $\wp_{L}\left(\left\{\phi^{k}\right\}\right) \subset c([0, \ell])$ for all $k \in \mathbb{N}$. We now claim that there 
exist some $i_{1} \in\{1,2, \ldots, p\}$ and $k \in\{0,1, \ldots, N+1\}$ such that

$$
\wp_{L}\left(\phi_{i_{1}}^{k}\right) \in c\left(\left[\gamma_{1}(\ell) / 2, \ell-\gamma_{1}(\ell) / 2\right]\right)
$$

where $\gamma_{1}: \mathbb{R}_{\geq 0} \rightarrow \mathbb{R}_{\geq 0}$ we borrow from Algorithm 1 . Now suppose that our claim is false. That implies that there exist two scalars $a, b$ satisfying $0 \leq a<\gamma_{1}(\ell) / 2$ and $\ell-\gamma_{1}(\ell) / 2<b \leq \ell$ and for all $k \in\{0,1, \ldots, N\}$ we have $\wp_{L}\left(\phi_{i}^{k}\right) \in c([0, a]) \cup c([b, \ell])$ for all $i$. Therefore, since $\mathbf{g} \in \mathbf{G}_{N}$, at some time $k \in\{0,1, \ldots, N\}$ there must exist a pair $(i, j)$ such that $\phi_{i}^{k} \in \mathbf{n}_{j}\left(\phi^{k}, \mathcal{A}_{g_{k}}\right)$ and one of the following holds:

- $\wp_{L}\left(\phi_{i}^{k}\right) \in c([0, a])$ and $\wp_{L}\left(\phi_{j}^{k}\right) \in c([b, \ell])$, or

- $\wp_{L}\left(\phi_{j}^{k}\right) \in c([0, a])$ and $\wp_{L}\left(\phi_{i}^{k}\right) \in c([b, \ell])$.

That implies conv $\wp_{L}\left(\mathbf{n}_{j}\left(\phi^{k}, \mathcal{A}_{g_{k}}\right)\right) \supset c([a, b])$. Thence it follows by Lemma 2 and (4) that $\wp_{L}\left(\phi_{j}^{k+1}\right) \in c\left(\left[\gamma_{1}(\ell), \ell-\right.\right.$ $\left.\left.\gamma_{1}(\ell)\right]\right)$, which poses a contradiction.

Now, let us recall that $\wp_{L}\left(\phi_{i}^{k}\right) \in \operatorname{conv} \wp_{L}\left(\mathbf{n}_{i}\left(\phi^{k}, \mathcal{A}_{g_{k}}\right)\right)$ for all $i$ and $k$. Therefore, if we combine (6) and Lemma 3, we can write

$$
\wp_{L}\left(\phi_{i_{1}}^{k}\right) \in c\left(\left[\gamma_{1}^{k}\left(\gamma_{1}(\ell) / 2\right), \ell-\gamma_{1}^{k}\left(\gamma_{1}(\ell) / 2\right)\right]\right)
$$

for $k \in \mathbb{N}_{\geq N+1}$. For compactness, let $h_{k}:=\gamma_{1}^{k}\left(\gamma_{1}(\ell) / 2\right)$. We can now make our second claim:

$$
\operatorname{diam} \wp_{L}\left(\left\{\phi^{(p-1)(N+1)}\right\}\right) \leq \ell-h_{(p-1)(N+1)} .
$$

Suppose not. Then there exist $i, j$ such that $\wp_{L}\left(\phi_{i}^{k}\right) \in$ $c\left(\left[0, h_{k}\right)\right)$ and $\wp_{L}\left(\phi_{j}^{k}\right) \in c\left(\left(\ell-h_{k}, \ell\right]\right)$ for all $k \in$ $\{0,1, \ldots,(p-1)(N+1)\}$. Observe that, due to Lemma 3 , if some agent $l$ satisfies $\wp_{L}\left(\phi_{l}^{k_{0}}\right) \in c\left(\left[h_{k_{0}}, \ell-h_{k_{0}}\right]\right)$ for some $k_{0}$ then $\wp_{L}\left(\phi_{l}^{k}\right) \in c\left(\left[h_{k}, \ell-h_{k}\right]\right)$ for all $k \in \mathbb{N}_{\geq k_{0}}$. Due to connectedness, there must be an agent $i_{2} \neq i_{1}$ such that conv $\wp_{L}\left(\mathbf{n}_{i_{2}}\left(\phi^{k}, \mathcal{A}_{g_{k}}\right)\right) \ni \wp_{L}\left(\phi_{i_{1}}^{k}\right)$ for some $k \in$ $\{N+1, N+2, \ldots, 2 N+1\}$. (Note that this does not necessarily imply $\mathbf{n}_{i_{2}}\left(\phi^{k}, \mathcal{A}_{g_{k}}\right) \ni \phi_{i_{1}}^{k}$.) Therefore, by (7) and Lemma 3 we can write

$$
\wp_{L}\left(\phi_{i_{2}}^{k}\right) \in c\left(\left[h_{k}, \ell-h_{k}\right]\right)
$$

for $k \in \mathbb{N}_{\geq 2 N+2}$. That is to say for all $k \in \mathbb{N}_{\geq 2 N+2}$ there will be at least two agents whose projections fall in $c\left(\left[h_{k}, \ell-h_{k}\right]\right)$. The generalization is straightforward and lets us to assert that for all $k \in \mathbb{N}_{\geq q(N+1)}$ there will be at least $q$ agents whose projections fall in $c\left(\left[h_{k}, \ell-h_{k}\right]\right)$. When $q=p-1$ we have a contradiction. Therefore (8) holds. Hence

$$
\begin{aligned}
\operatorname{diam} \wp_{L}\left(\left\{\phi^{(p-1)(N+1)}\right\}\right) & \leq \ell-\gamma_{1}^{(p-1)(N+1)}\left(\gamma_{1}(\ell) / 2\right) \\
& \leq \gamma_{3}\left(\operatorname{diam} \wp_{L}\left(\left\{\phi^{0}\right\}\right)\right) .
\end{aligned}
$$

Going one step further we can write

$$
\operatorname{diam} \wp_{L}\left(\left\{\phi^{m(p-1)(N+1)}\right\}\right) \leq \gamma_{3}^{m}\left(\operatorname{diam} \wp_{L}\left(\left\{\phi^{0}\right\}\right)\right)
$$

for all $m \in \mathbb{N}$. All that is left is to combine (5) with (9).

Theorem 3 Let $C$ be a convex subset of $\mathbb{X}, \omega \leq \mathrm{id} / 2$ be a class- $\mathcal{K}$ function, and $N \in \mathbb{N}$. Suppose that system (1) is contractive on $C$ with $\omega$. Then for all $\{\mathbf{x}\} \subset C$ and $\mathbf{g} \in \mathbf{G}_{N}$ we have

$$
\operatorname{diam}\{\Phi(k, \mathbf{x}, \mathbf{g})\} \leq \beta_{(\omega, p, N)}(\operatorname{diam}\{\mathbf{x}\}, k)
$$

for all $k \in \mathbb{N}$, where function $\beta_{(\omega, p, N)}$ is constructed according to Algorithm 1.

Proof. Let us be given $\{\mathbf{x}\} \subset C, \mathbf{g} \in \mathbf{G}_{N}$, and $k \in$ $\mathbb{N}$. Let $x, y \in\{\Phi(k, \mathbf{x}, \mathbf{g})\}$ be such that $d(x, y)=$ $\operatorname{diam}\{\Phi(k, \mathbf{x}, \mathbf{g})\}$. Then let $L \in \mathcal{L}_{\mathbb{X}}$ be a geodesic line such that $x, y \in L$. By Lemma 4 and remembering that $\beta_{(\omega, p, N)}$ is a class- $\mathcal{K} \mathcal{L}$ function and that $\wp_{L}$ does not increase distances, we can write

$$
\begin{aligned}
\operatorname{diam}\{\Phi(k, \mathbf{x}, \mathbf{g})\} & =\operatorname{diam}_{\wp_{L}}(\{\Phi(k, \mathbf{x}, \mathbf{g})\}) \\
& \leq \beta_{(\omega, p, N)}\left(\operatorname{diam} \wp_{L}(\{\mathbf{x}\}), k\right) \\
& \leq \beta_{(\omega, p, N)}(\operatorname{diam}\{\mathbf{x}\}, k) .
\end{aligned}
$$

Hence the result.

The convergence shown in Theorem 3 becomes exponential when the contraction is characterized by a linear function. The following corollary formalizes this assertion.

Corollary 1 Let $C$ be a convex subset of $\mathbb{X}, w \in(0,1 / 2]$, and $N \in \mathbb{N}$. Suppose that system (1) is contractive on $C$ with $w \cdot$ id. Then for all $\{\mathbf{x}\} \subset C$ and $\mathbf{g} \in \mathbf{G}_{N}$ we have

$$
\operatorname{diam}\{\Phi(k, \mathbf{x}, \mathbf{g})\} \leq M \sigma^{k} \operatorname{diam}\{\mathbf{x}\}
$$

for all $k \in \mathbb{N}$, where pair $(M, \sigma)$ is obtained from Algorithm 2.

We end the section with a proof of Theorem 2 .

Proof of Theorem 2. Stability and boundedness follow from Theorem 1, attractivity from Theorem 3.

\section{LINEAR EXAMPLE}

In this section we study a linear system (cf. [9]) and show that it is contractive uniformly with a single linear class- $\mathcal{K}$ function which can be explicitly computed.

Let $w_{i j}: \mathcal{G} \rightarrow \mathbb{R}_{\geq 0}$, defined for each $i, j \in\{1,2, \ldots, p\}$, be a weight map satisfying the following conditions:

1) $w_{i j}(g)=0$ if $j \notin\left\{l: \mathbf{x}_{l} \in \mathbf{n}_{i}\left(\mathbf{x}, \mathcal{A}_{g}\right)\right\}$,

2) $w_{i j}(g) \geq w_{\min }>0$ if $j \in\left\{l: \mathbf{x}_{l} \in \mathbf{n}_{i}\left(\mathbf{x}, \mathcal{A}_{g}\right)\right\}$,

3) $\sum_{j} w_{i j}(g)=1$.

Proposition 5 Consider system (1). Let $\mathbb{X}=\mathbb{R}^{n}, d: \mathbb{R}^{n} \times$ $\mathbb{R}^{n} \rightarrow \mathbb{R}_{\geq 0}$ be the standard Euclidean metric, i.e. $d(x, y)=$ $|x-y|$ for $x, y \in \mathbb{R}^{n}$, and the righthand side obeys

$$
\mathbf{f}_{i}(\mathbf{x}, g)=\sum_{j} w_{i j}(g) \cdot \mathbf{x}_{j}
$$

for all $i \in\{1,2, \ldots, p\}$. Then system (1) is contractive on $\mathbb{R}^{n}$ with $w_{\text {min }} \cdot$ id.

Proof. Given $\mathbf{x}, i, g$, and a line $L \subset \mathbb{R}^{n}$ we can write

$$
\wp_{L}\left(\mathbf{f}_{i}(\mathbf{x}, g)\right)=\sum_{j} w_{i j}(g) \cdot \wp_{L}\left(\mathbf{x}_{j}\right) .
$$


Note that projection point $\wp_{L}\left(\mathbf{f}_{i}(\mathbf{x}, g)\right)$ lies on the line segment $\operatorname{conv} \wp_{L}\left(\mathbf{n}_{i}\left(\mathbf{x}, \mathcal{A}_{g}\right)\right)$. Due to (10), point $\wp_{L}\left(\mathbf{f}_{i}(\mathbf{x}, g)\right)$ cannot be closer to either of the endpoints of the line segment $\operatorname{conv} \wp_{L}\left(\mathbf{n}_{i}\left(\mathbf{x}, \mathcal{A}_{g}\right)\right)$ than $w_{\min } \cdot \operatorname{diam} \wp_{L}\left(\mathbf{n}_{i}\left(\mathbf{x}, \mathcal{A}_{g}\right)\right)$. Therefore we have

$$
\wp_{L}\left(\mathbf{f}_{i}(\mathbf{x}, g)\right) \subset \overline{\operatorname{cont}}\left(\operatorname{conv} \mathbf{n}_{i}\left(\mathbf{x}, \mathcal{A}_{g}\right), w_{\min } \cdot \mathrm{id}, L\right)
$$

which implies

$$
\mathbf{f}_{i}(\mathbf{x}, g) \in \overline{\operatorname{cont}}\left(\operatorname{conv} \mathbf{n}_{i}\left(\mathbf{x}, \mathcal{A}_{g}\right), w_{\min } \cdot \mathrm{id}, L\right) .
$$

Since line $L$ is arbitrary we can write

$$
\mathbf{f}_{i}(\mathbf{x}, g) \in \operatorname{cont}\left(\operatorname{conv} \mathbf{n}_{i}\left(\mathbf{x}, \mathcal{A}_{g}\right), w_{\min } \cdot \mathrm{id}\right) .
$$

Hence the result.

Remark 1 For the case considered in Proposition 5, Standing Assumption 1 comes for free.

Corollary 2 Consider system (1). Let $\mathbb{X}=\mathbb{R}^{n}, d: \mathbb{R}^{n} \times$ $\mathbb{R}^{n} \rightarrow \mathbb{R}_{\geq 0}$ be the standard Euclidean metric, i.e. $d(x, y)=$ $|x-y|$ for $x, y \in \mathbb{R}^{n}$, and for all $i \in\{1,2, \ldots, p\}$

$$
\mathbf{f}_{i}(\mathbf{x}, g)=\sum_{j} w_{i j}(g) \cdot \mathbf{x}_{j} \text {. }
$$

Then for all $\{\mathbf{x}\} \subset \mathbb{R}^{n}$ and $\mathbf{g} \in \mathbf{G}_{N}$

$$
\operatorname{diam}\{\Phi(k, \mathbf{x}, \mathbf{g})\} \leq M \sigma^{k} \operatorname{diam}\{\mathbf{x}\}
$$

for all $k \in \mathbb{N}$, where pair $(M, \sigma)$ is obtained from Algorithm 2 with $w=w_{\min }$.

\section{LOGLINEAR EXAMPLE}

Another example where the contraction can uniformly be characterized by a single linear class- $\mathcal{K}$ function is a loglinear system. This rather academic example is obtained from the system considered in Section VI via an exponential transformation. That is the reason we call it a loglinear system, for it becomes linear under logarithm. In a linear system, where the state of an agent at the next time step is a convex combination of the current states of itself and its neighbors, evolving toward a consensus is closely related to computing (an) arithmetic mean of the individual agents. From that respect, in a loglinear system, what is being computed is (a) geometric mean. Below, we introduce some notation. Then we present the counterparts of the results of Section VI where we resort to the weight function defined therein. The proofs will be omitted.

Given two points $x, y$ in $\mathbb{R}_{>0}^{n}$, let $x / y$ denote the elementwise division, i.e. $x / y:=\left(x_{1} / y_{1}, x_{2} / y_{2}, \ldots, x_{n} / y_{n}\right)$, and $x y$ denote the elementwise product. Likewise, given $a \in \mathbb{R}$, let $x^{a}$ denote elementwise exponentiation, i.e. $x^{a}:=\left(x_{1}^{a}, x_{2}^{a}, \ldots, x_{n}^{a}\right)$. Finally, we let $\ln (x):=$ $\left(\ln \left(x_{1}\right), \ln \left(x_{2}\right), \ldots, \ln \left(x_{n}\right)\right)$.

Proposition 6 Consider system (1). Let $\mathbb{X}=\mathbb{R}_{>0}^{n}, d: \mathbb{R}_{>0}^{n} \times$ $\mathbb{R}_{>0}^{n} \rightarrow \mathbb{R}_{\geq 0}$ be defined as $d(x, y):=|\ln (x / y)|$ for $x, y \in$ $\mathbb{R}_{>0}^{n}$, and the righthand side obeys

$$
\mathbf{f}_{i}(\mathbf{x}, g)=\prod_{j} \mathbf{x}_{j}^{w_{i j}(g)}
$$

for all $i \in\{1,2, \ldots, p\}$. Then system (1) is contractive on $\mathbb{R}_{>0}^{n}$ with $w_{\text {min }} \cdot$ id.

Remark 2 For the case considered in Proposition 6, Standing Assumption 1 comes for free.

Corollary 3 Consider system (1). Let $\mathbb{X}=\mathbb{R}_{>0}^{n}, d: \mathbb{R}_{>0}^{n} \times$ $\mathbb{R}_{>0}^{n} \rightarrow \mathbb{R}_{\geq 0}$ be defined as $d(x, y):=|\ln (x / y)|$ for $x, y \in$ $\mathbb{R}_{>0}^{n}$, and for all $i \in\{1,2, \ldots, p\}$

$$
\mathbf{f}_{i}(\mathbf{x}, g)=\prod_{j} \mathbf{x}_{j}^{w_{i j}(g)} .
$$

Then for all $\{\mathbf{x}\} \subset \mathbb{R}_{>0}^{n}$ and $\mathbf{g} \in \mathbf{G}_{N}$

$$
\operatorname{diam}\{\Phi(k, \mathbf{x}, \mathbf{g})\} \leq M \sigma^{k} \operatorname{diam}\{\mathbf{x}\}
$$

for all $k \in \mathbb{N}$, where pair $(M, \sigma)$ is obtained from Algorithm 2 with $w=w_{\min }$.

\section{CONCLUSION}

In this paper we have studied discrete-time multi-agent systems and obtained explicit convergence (to a consensus) rates in terms of the number of agents, the length of the interval over which the communication graph is connected, and the class- $\mathcal{K}$ function characterizing the contraction of the convex sets that should include the values that the agents' transition maps take. We have considered convexity in metric spaces more general than $\mathbb{R}^{n}$, where a set is considered convex if it contains all the geodesic segments associated to its points. (By geodesic segment we mean the shortest path between two points with respect to the distance function of interest.)

\section{REFERENCES}

[1] T. Vicsek, A. Czirok, E. Ben-Jacob, I. Cohen, and O. Schochet, "Novel type of phase transitions in a system of self-driven particles," Physical Review Letters, vol. 75(6), pp. 1226-1229, 1995.

[2] J. Fax and R. Murray, "Information flow and cooperative control of vehicle formations," IEEE Transactions on Automatic Control, vol. 49(9), pp. 1465-1476, 2004.

[3] R. Sepulchre, D. Paley, and N. Leonard, "Collective motion and oscillator synchronization," in Cooperative Control (Lecture Notes in Control and Information Sciences), V. Kumar, N. Leonard, and A. Morse, Eds. Springer-Verlag, 2004, vol. 309, pp. 189-205.

[4] L. Xiao, S. Boyd, and S. Lall, "A scheme for asynchronuous distributed sensor fusion based on average consensus," in Proc. of the 4th International Symposium on Information Processing in Sensor Networks, 2005, pp. 63-70.

[5] R. Olfati-Saber, J. Fax, and R. Murray, "Consensus and cooperation in networked multi-agent systems," Proceedings of the IEEE, vol. 95(1), pp. 1-17, 2007.

[6] L. Moreau, "Stability of multi-agent systems with time-dependent communication links," IEEE Transactions on Automatic Control, vol. 50(2), pp. 169-182, 2005.

[7] D. Angeli and P.-A. Bliman, "Stability of leaderless discrete-time multi-agent systems," Mathematics of Control, Signals \& Systems, vol. 18(4), pp. 293-322, 2006.

[8] S. Tuna and R. Sepulchre, "Quantitative convergence analysis of multiagent systems," in Proc. of the 7th IFAC Symposium on Nonlinear Control Systems, 2007, pp. 236-241.

[9] V. Blondel, J. Hendrickx, A. Olshevsky, and J. Tsitsiklis, "Convergence in multiagent coordination, consensus, and flocking," in Proc. of the 44th IEEE Conference on Decision and Control, 2005, pp. 29963000.

[10] M. Bridson and A. Haefliger, Metric Spaces of Non-Positive Curvature. Springer-Verlag, 1999. 